\title{
Linking Length Scales and Modalities with Integrated, Correlative Microscopy
}

\author{
Jeff Gelb ${ }^{1}$, Will Harris ${ }^{1}$, Lorenz Lechner ${ }^{1}$ and Arno Merkle ${ }^{1}$ \\ 1. Carl Zeiss X-Ray Microscopy, Inc., Pleasanton, CA, USA
}

Modern microscopy labs are typically outfitted with a suite of instruments, capable of capturing data across a range of length scales, from the centimeters to the sub-nanometers. These imaging instruments are often complimented by analytical techniques, such as spectroscopic chemical characterization platforms, and are designed to produce a comprehensive depiction of the material under investigation. However, often these techniques exist independently from each other - a specimen may be imaged on a light microscope, or an SEM, or an X-ray microscope, but rarely are the instruments used together in concert to effectively utilize the unique strengths of each tool. This is generally not due to lack of interest, but, rather, due to the technology challenges associated with data registration, image alignment, and microscope cross-calibration, which impose tactical constraints that make correlative microscopy a very challenging task.

In recent years, the microscopy industry has begun to embrace the need for an integrated approach, and has been working closely with leading researchers to develop an effective, easy-to-use approach for combining data from multiple modalities. Here, we present a unique advancement enabling correlative microscopy, which uses a centralized software platform to pull together data from light-, electron-, and $\mathrm{X}$-ray microscopy (XRM). Beyond just correlating the various datasets, the approach allows data from one technique to be used to drive the hardware in another technique, facilitating easy transfer of information between the suite of available microscopes and the operator.

In support of this development, we will present the results from several unique case studies, where the integrated, correlative microscopy approach has elucidated interesting features about the specimens being studied. In the first example, a 7075 Aluminum Alloy was investigated across a range of length scales, utilizing both X-ray and electron microscopy (FIB-SEM). Initial surveys with the XRM revealed the large-scale heterogeneity of the alloy and enabled the bulk inclusions to be virtually extracted with sub-micron resolution. Using a targeted region identified within the XRM volume, the FIB was then instructed to isolate and reveal that region, which showed several nano-scale precipitates as well as local voids. For the second example, a magnesium alloy was imaged in corrosive conditions with the XRM and, subsequently, with nano-scale XRM to reveal the byproducts of the corrosion reaction. This 4D study was performed over the course of $\sim 8$ hours and enabled a small region to be identified for postmortem analysis with the FIB-SEM. Through FIB cross-sectioning and 3D FIB tomography, the precise nature of the corrosion reaction was visualized in 3D, which provided a unique insight into the structural components of corrosion within this particular material. Finally, an 18650 Li-ion battery was sectioned and a small piece extracted for correlative XRM-SEM analysis. The XRM results revealed apparent local chemical inhomogeneities, which were surfaced with the FIB and characterized with energydispersive X-ray spectroscopy (EDS). From the EDS results, the identity of the inhomogeneities were confirmed to be La, which was an unexpected material to find inside the battery.

References:

[1] A P Merkle and J Gelb, Microscopy Today (2013) pp. 10-15.

[2] A P Merkle et al, Microscopy and Analysis (2014) pp. S10-S13. 


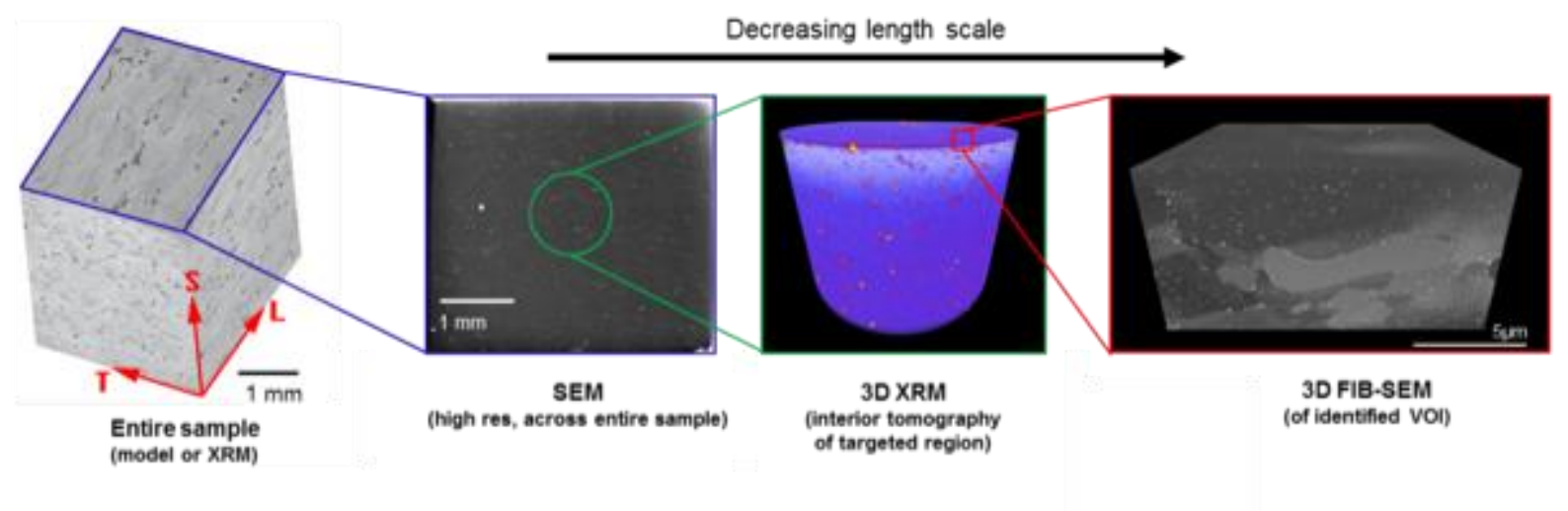

Figure 1. A correlative microscopy workflow uses each technique for its unique strength, using the Xray microscopes (XRMs), for example, to survey a material and capture the time evolution of the microstructure. These results may be used for further imaging with the FIB-SEM, to produce targeted cross-section, spectroscopic, or 3D tomographic results in a targeting location, as shown here.

\section{Decreasing length scale}

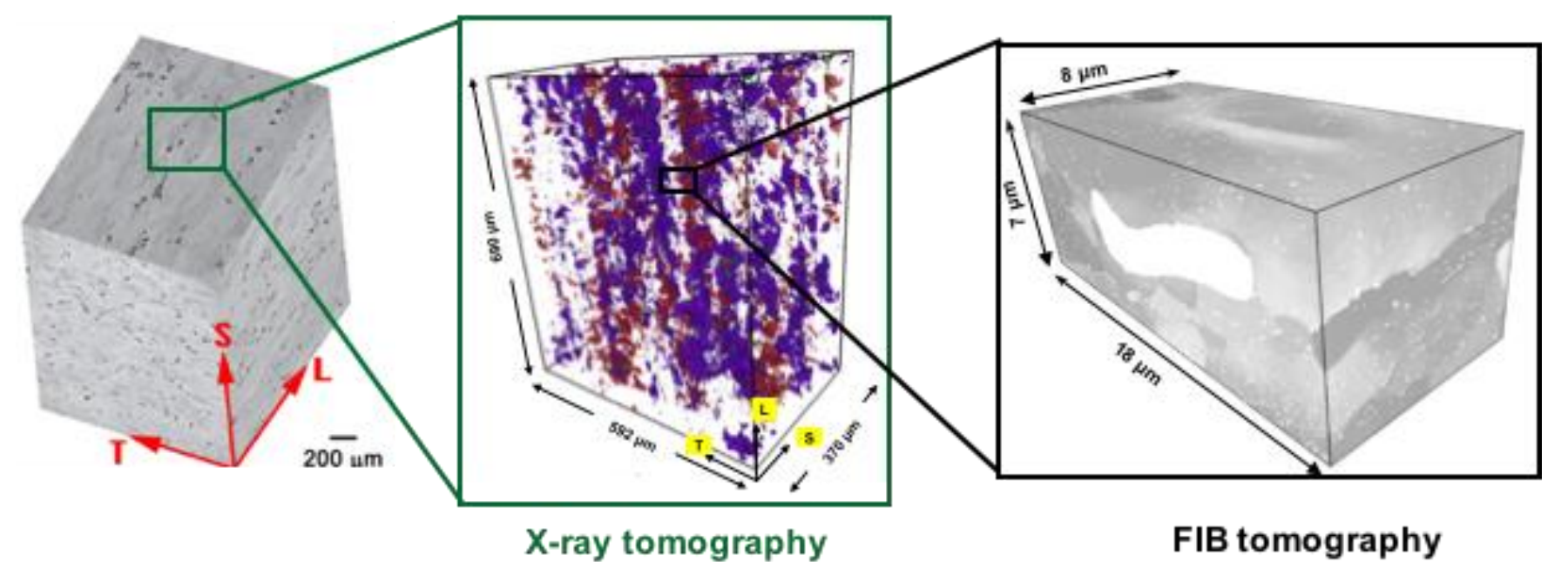

Figure 2. Applying this technique to a 7075 aluminum alloy, the 3D XRM volumes were used to identify the bulk inclusions and to localize a region of interest for the FIB. These results were aligned with the SEM and used to drive the FIB to the identified region for high-resolution 3D analysis of the nano-scale precipitates and local voids. 